\title{
Reduced cortical GABA and glutamate in high schizotypy
}

\author{
Petya Kozhuharova' ${ }^{1} \cdot$ Andreea O. Diaconescu ${ }^{2,3,4,5} \cdot$ Paul Allen $^{1,6}$
}

Received: 24 November 2020 / Accepted: 2 May 2021 / Published online: 19 June 2021

(c) Crown 2021

\begin{abstract}
Rationale Abnormal functioning of the inhibitory gamma-aminobutyric acid (GABA) and excitatory (glutamate) systems is proposed to play a role in the development of schizophrenia spectrum disorder. Although results are mixed, previous $1 \mathrm{H}$-magnetic resonance spectroscopy (MRS) studies in schizophrenia and clinical high-risk samples report these metabolites are altered in comparison to healthy controls. Currently, however, there are few studies of these metabolites in schizotypy samples, a personality dimension associated with the experience of schizophrenia and psychosis-like symptoms.

Objectives We investigated if GABA and glutamate metabolite concentrations are altered in people with high schizotypy. We also explored the relationship between resilience to stress, GABA metabolite concentrations and schizotypy.

Methods We used MRS to examine GABA and glutamate levels in the medial prefrontal cortex in people with low and high schizotypy traits as assessed with the Schizotypal Personality Questionnaire. Resilience to stress was assessed using the Connor-Davidson Resilience Scale.

Results Compared to individuals with low schizotypy traits, high schizotypy individuals showed lower cortical prefrontal $\operatorname{GABA}\left(F(1,38)=5.18, p=0.03, \eta^{2}=0.09\right)$ and glutamate metabolite levels $\left(F(1,49)=6.25, p=0.02, \eta^{2}=0.02\right)$. Furthermore, participants with high GABA and high resilience levels were significantly more likely to be in the low schizotypy group than participants with low GABA and high resilience or high GABA and low resilience (95\% CI 1.07-1.34, $p<.001$ ). Conclusions These findings demonstrate that subclinical schizotypal traits are associated with abnormal functioning of both inhibitory and excitatory systems and suggest that these transmitters are implicated in a personality trait believed to be on a continuum with psychosis.
\end{abstract}

Keywords Glutamate $\cdot$ GABA $\cdot$ Spectroscopy $\cdot$ Schizotypy $\cdot$ Schizophrenia $\cdot$ Risk $\cdot$ Cortical $\cdot$ Metabolites

This article belongs to a Special Issue on CNS imaging

Petya Kozhuharova

kozhuhap@roehampton.ac.uk

1 Centre for Cognition, Neuroscience and Neuroimaging, Department of Psychology, University of Roehampton, Holybourne Ave, Roehampton, London SW15 4JD, UK

2 Department of Psychiatry, Brain and Therapeutics, Krembil Centre for Neuroinformatics, CAMH, Toronto, Canada

3 Department of Psychiatry, University of Toronto, Toronto, ON, Canada

4 Institute of Medical Sciences, University of Toronto, Toronto, ON, Canada

5 Department of Psychology, University of Toronto, Toronto, ON, Canada

6 Department of Psychosis Studies, Institute of Psychiatry, Psychology and Neuroscience, King's College London, London, UK

\section{Introduction}

There is a growing consensus that psychosis exists on a continuum ranging from subclinical psychotic-like experiences in the general population to full-blown psychotic symptoms in clinical samples (Linscott and Van Os 2013). Psychoticlike experiences in healthy people, commonly referred to as schizotypy, represent a latent personality organization reflecting an underlying vulnerability to developing schizophrenia spectrum disorders (Barrantes-Vidal et al. 2014). Investigating schizotypal traits in non-clinical samples may provide information about neurobiological alterations underlying psychosis-like symptoms (phenotype) in the absence of confounding factors such as antipsychotic medication.

Schizotypal personality traits are qualitatively similar, but less severe than the symptoms seen in patients with schizophrenia. Relative to patients with schizophrenia, individuals scoring high on schizotypy trait measures show similar, 
albeit less severe, deficits in cognition and aberrations of perception, thought and beliefs. High schizotypy has also been linked with brain structural and functional abnormalities relative to control groups (Ettinger et al. 2014). However, fewer studies have investigated if there are neurochemical alterations in schizotypy populations similar to those reported in schizophrenia (Egerton et al. 2017) and predicted by animal models to influence psychosis symptomatology (Du and Grace 2013).

Whilst several animal studies show the significance of dopaminergic functions in psychosis risk/symptomatology (Du and Grace 2013; Grace 2010; Goto and Grace 2006), there have only been few studies examining GABAergic and/or glutamate function in schizotypy populations. The investigation of GABAergic and glutamatergic function is important because evidence from animal models and post-mortem studies of psychosis suggests that dysregulated excitatory and inhibitory neurotransmission plays an important role in the development of schizophrenia-like symptoms (Du and Grace 2013; Grace 2010; Goto and Grace 2006). The pre-clinical methylazoxymethanol acetate (MAM) rodent model of psychosis shows reduced parvalbumin expression in MAM-treated rats that may be linked to schizophrenia-like pathology (Gastambide et al. 2012). In particular, reduced parvalbumin expression may impact on prefrontal cortex (PFC) GABAergic interneurons that are known to be decreased in schizophrenia populations (Akbarian et al. 1995; Lewis et al. 2005). The MAM model also proposes dysfunction of the glutamatergic system as a possible mechanism that increases risk for psychosis (Marsman et al. 2013). The glutamatergic system is believed to affect synaptic plasticity and cortical microcircuitry, in particular (N-methyl-D-aspartate) NMDA receptor signalling (Merritt et al. 2016). Furthermore, the MAM model of psychosis emphasises a link between disrupted GABAergic and glutamatergic function and dysregulation of subcortical dopaminergic signalling (Grace 2016, 2010) that is thought to underlie positive symptoms such as delusions.

Consistent with pre-clinical work, studies in human subjects with a diagnosis of schizophrenia (Marsman et al. 2013; Merritt et al. 2016) report altered GABAergic and glutamatergic function relative to healthy control groups across a range of cortical and subcortical regions. However, a meta-analysis of 1H-MRS studies in schizophrenia and high-risk groups reports no overall difference in $\mathrm{mPFC}$ GABA metabolite concentrations relative to healthy controls (Egerton et al. 2017). Thus, it is unclear if mPFC GABA levels are altered in people with schizophrenia or in those with a high risk for the illness. Moreover, pharmacological challenge studies in humans report that the administration of NMDA receptor antagonists, such as ketamine and phencyclidine (PCP), induces symptoms that mimic the positive and negative symptoms seen in schizophrenia (Harrison and
Weinberger 2005; Krystal et al. 1994; Moghaddam et al. 1997).

Crucially, however, studies investigating GABA and glutamate metabolite concentrations in high schizotypy samples are limited. Such studies are important if we are to better understand the role of (perturbed) inhibitory and excitatory neurotransmission across the psychosis continuum. One previous study investigating glutamate levels in individuals with high positive schizotypy traits reported no differences in metabolite concentration levels in the anterior cingulate cortex relative to a low positive schizotypy control group. There was however an interaction effect such that glutamate levels were negatively associated with the degree of cortical activation in response to emotional pictures in the striatum and the mPFC (Modinos et al. 2018). These preliminary findings suggest that cortical glutamate levels might be perturbed in high (positive) schizotypy in the context of affective function.

Given the paucity of $1 \mathrm{H}$-magnetic resonance spectroscopy (MRS) studies in schizotypy populations, in the current study, we first sought to investigate GABA and glutamate metabolite concentrations in a sample of high schizotypy participants, relative to a low schizotypy control group. We used MRS with a voxel located in the medial PFC as preclinical models implicate this region in the neuropathology of psychosis and psychosis risk. Moreover, a number of previous MRS studies in CHR and schizophrenia samples have investigated this region and reported altered metabolite concentrations in the mPFC (Becker et al. 2003; Modinos et al. 2017; Mailly et al. 2013). Given that MRS studies in schizophrenia cohorts have reported both increased and decreased GABA and glutamate metabolite concentrations in prefrontal regions (Marsman et al. 2013; Merritt et al. 2016), we cannot predict a direction of this effect and instead predicted that relative to low schizotypy participants, high schizotypy participants will show altered GABA and glutamate metabolite concentration in the mPFC.

Our second aim was to explore if the relationship between cortical GABA metabolite concentrations and schizotypy was affected by resilience (to stress). Work in animals shows that stress, induced by injecting rats with corticosterone, leads to a decrease of mRNA for GAD67, the enzyme that synthesises GABA (Stone et al. 2001; Deslauriers et al. 2013; Giovanoli et al. 2013). Moreover, animal models of schizophrenia indicate that the GABA system is strongly influenced by environmental stress (Zhang et al. 2010; Guidotti et al. 2011), and that in humans, stress exposure in adolescence can lead to prodromal psychotic symptoms (Zimmerman et al. 2013). Taken together, these findings suggest that stress may affect GABAergic interneurons leading to the emergence of schizophrenia-like symptoms (Powell et al. 2012). However, to date, no study has investigated the relationship between stress/resilience, GABA 
metabolite concentrations, and schizotypy. To address this, we explored if the relationship between resilience levels and GABA metabolite concentrations differed across low and high schizotypy groups. We hypothesised that mPFC GABA metabolite concentrations would interact with resilience scores to predict SPQ group membership (high vs low), and that low and high schizotypy groups would show a different relationship between GABA metabolite concentrations and resilience levels.

\section{Methods}

\section{Participants}

One thousand three hundred forty-two participants responded to an online survey advertised via social media and were pre-screened using the Schizotypy Personality Questionnaire (SPQ; Raine 1991) and the Marlowe-Crowne Social Desirability Scale (SDS; Fischer and Fick 1993). All participants that took part in the MRI study were recruited from the student population of the Royal Holloway University of London. Exclusion criteria were defined as the presence of contraindicators for MRI scanning (presence of metal, etc.), current use of prescribed medication for neuropsychiatric disorders or history of neuropsychiatric disorders, current use or history of illicit substance misuse. These criteria were assessed via self-report and pre-screening for MRI scanning. The SDS questionnaire was used to exclude participants that give mainly socially desirable answers; thus, only subjects scoring 8 or higher on this measure were excluded to control for socially desirable responding (Fischer and Fick 1993).

Subjects were invited to take part in the study based on their SPQ score. The aim of the study was to recruit the bottom and top 10\% (decile) of the schizotypy continuum (SPQ distribution); thus, individuals scoring below 12 and above 41 points on the SPQ were invited to take part (as informed by previous research; Raine 1991). The SPQ provides an overall measure of individual differences in schizotypal personality traits and can be reduced to three latent dimensions (positive, disorganised and negative; Raine 1991), mimicking the symptom clusters of schizophrenia and clinical highrisk states. The final sample included 27 participants in the high schizotypy group (HS; 17 females, age range 18-22, $\mathrm{M}=19.25, \mathrm{SD}=1.05)$ and 26 participants in the low schizotypy group (LS; 19 females, age range $18-27, \mathrm{M}=20.38$, $\mathrm{SD}=2.02)$. The sample size was based on a generic effect size (0.6 to 0.8 ) calculation that would allow us to detect a medium effect based on $80 \%$ power and an alpha $=0.05$ in our sample.

Ethical approval for the study was obtained from the University of Roehampton's Ethics Committee and all participants provided informed written consent before initiating any study procedures. Participants were compensated for their time ( $£ 40$ cash payment and a high-resolution anatomical scan of their brain).

Participants also completed demographic and substance use measures and the 25-item Connor-Davidson Resilience Scale (CD-RISC; Campbell-Sills and Stein 2007) to measure their resilience levels. The CD-RISC scale was developed to measure the ability to cope with adversity, with higher scores indicating greater resilience. Higher CD-RISC scores indicate higher levels of resilience and an increased ability to cope with stress (Campbell-Sills and Stein 2007). On the day of MRI scanning, participants completed a validated short version of the Wechsler abbreviated scale of intelligence (WASI II) to assess intellectual ability (IQ). We also used the Brief Symptom Inventory-Short Form (SF, Furlanetto et al. 2005) and Spielberger State Trait Anxiety Inventory (Grös et al. 2007) to measure depressive and anxiety traits in participants.

\section{MRI acquisition}

All MRI scans were acquired on a 3 T Siemens Magnetom TIM Trio scanner using a 32-channel head coil. Structural T1-weighted magnetization-prepared rapid acquisition gradient echo (MP RAGE) images were acquired with a spatial resolution of $1 \mathrm{~mm} \times 1 \mathrm{~mm} \times 1 \mathrm{~mm}$, in plane resolution of $256 \times 256 \times 176$ continuous slices, TR of 1900 s and scanning time of approximately $5 \mathrm{~min}$. T1 MP RAGE scans were acquired for localization of the spectroscopy voxel placement and were subsequently segmented into tissue maps to allow volume correction for grey and white matter as well as CSF within the $\mathrm{mPFC}$ voxel, i.e. partial volume tissue contamination.

\section{H-MRS data acquisition and analysis}

$1 \mathrm{H}-\mathrm{MRS}$ in vivo spectra were acquired from a $20 \times 20 \times 20 \mathrm{~mm}$ voxel located in the bilateral medial PFC during rest (Fig. 1). The reasoning for choosing a medial PFC position was two-fold. First, lateral voxels can be harder to place due to tissue boundaries. Second, the medial PFC has shown abnormal GABA levels in schizophrenia patients in a number of previous studies and is one of the most commonly used MRS voxel placements for psychosis populations (Egerton et al. 2017; Modinos et al. 2017; Stone et al. 2009).

The voxel was positioned manually by reference to an axial T1-weighted gradient echo image (Fig. 1). Spectra were acquired using spin echo full intensity-acquired localized spectroscopy (SPECIAL; Mlynárik et al. 2006). 1H-MRS sequence was acquired with water suppression (TR $3000 \mathrm{~ms}$, TE $8.5 \mathrm{~ms}$, phase cycle auto, 192 averages from 


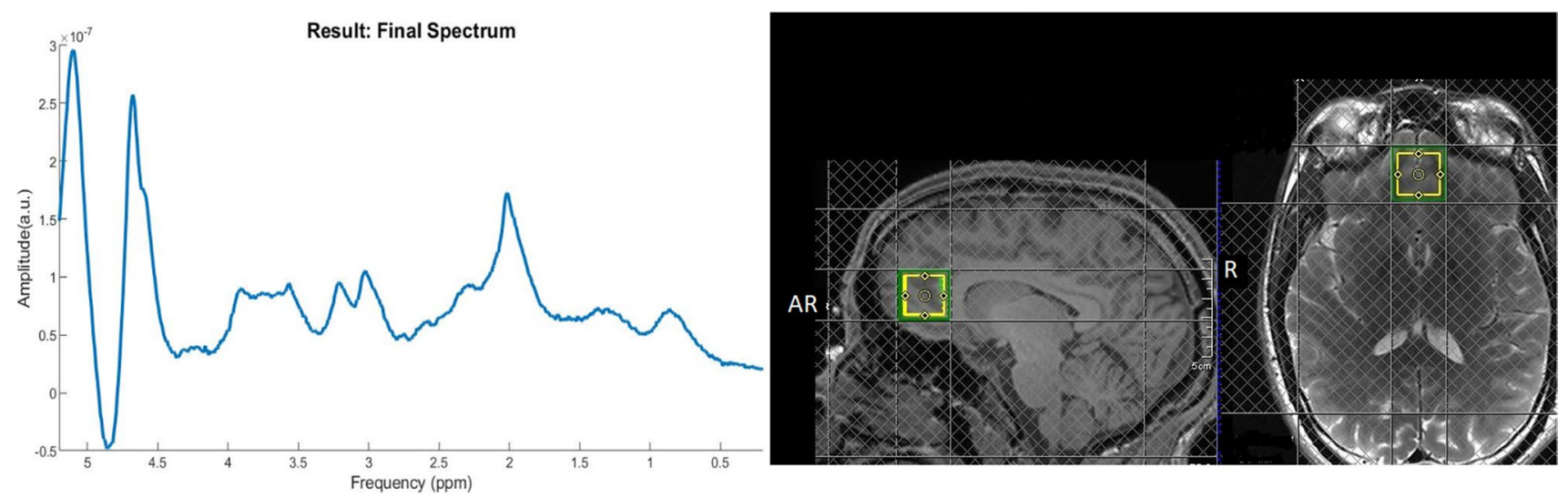

Fig. 1 Voxel placement in the mPFC shown on an example participant (sagittal and axial orientation) and a representative 1H-MRS final spectra included from LCModel

the right PFC voxel) in each participant (Godlewska et al. 2015). Water unsuppressed spectra (16 averages) were also acquired. Six outer volume suppression slabs were applied (one on each side at $5 \mathrm{~mm}$ from the edge of the cubic voxel) to suppress signals originating from outside the volume of interest and to minimize motion-related image-selected in vivo spectroscopy subtraction artefacts. Spectra were analysed using LCModel 6.3-1 $\mathrm{N}$ with the basis set consisting of 19 simulated basis spectra: alanine (Ala), ascorbate (Asc), aspartate (Asp), creatine (Cr), $\gamma$-aminobutyric acid (GABA), glucose (Glc), glutamine (Gln), glutamate (Glu), glycine (Gly), glutathione (GSH), glycerophosphocholine (GPC), phosphocholine (PCh), lactate (Lac), myo-inositol (mI), N-acetylaspartate (NAA), N-acetyl aspartate glutamate (NAAG), phosphorylethanolamine (PE), scyllo-inositol (Scyllo) and taurine (Tau).

The basis set was simulated using FID-A [61] for $\mathrm{TE}=8.5 \mathrm{~ms}$, magnetic field strength $=3 \mathrm{~T}$ and assuming ideal RF pulses. We excluded spectra with Cramer-Rao lower bounds (CRLB) $>20 \%$ as reported by LCModel. Line widths and signal-to-noise ratios were calculated by LCModel for both LS and HS groups (see Results). All spectra had a line width $<8 \mathrm{~Hz}$ and an SNR $>40$ (Godlewska et al. 2015). Following these quality control checks, 8 participants from the HS group and 5 participants from the LS group were excluded due to CLRB ratios for GABA $>20 \%$. Thus, the reported results for GABA are from 40 subjects in total (19 HS and $21 \mathrm{LS}$ ). For analysis of glutamate, quality control checks indicated that 2 subjects from the HS group had to be excluded due to CLRB ratios for Glu $>20 \%$, resulting in a total sample of $25 \mathrm{HS}$ and $26 \mathrm{LS}$ individuals from the analysis of glutamate.

Metabolite levels have been shown to depend on the amount of cerebrospinal fluid (CSF), gray (GM) and white matter (WM) within the voxel (Srinivasn et al. 2006), and inter-individual differences in cortical gray matter (Huster et al. 2007). To account for these confounds, we used the T1-weighted anatomical images to estimate the gray and white matter content of the mPFC voxel in which the 1H-MRS measures were acquired using FSL FAST segmentation (Zhang et al. 2001). For every subject, tissue masks for CSF, GM and WM were created using FSL and the following procedure: registering the tissue template in MNI space to native space using FSL prior tissue probability maps, finding overlap between the tissue probability maps and the tissue template created in the previous step, applying a 0.4 threshold and binarizing the tissue templates, and finally generating the tissue masks by applying the prior in native space to the binarized tissue probability map.

Following this, for every individual subject, the placement of the MRS voxel was applied as a $20 \times 20 \times 20$ mask (individually measured for each subject based on 1H-MRS acquisition) to the three respective tissue maps providing a segmentation quantity for each tissue in the specific 1H-MRS voxel placement. CSF, GM and WM were then accounted for in the expression of Glu and GABA levels using LCModel (Gasparovic et al. 2006); corrected metabolite levels are referred to as Glu-Corr and GABA-Corr using the formula Glu-Corr $=\left(\mathrm{Glu}^{*}(43,300 * \mathrm{GMV}+35,880 * \mathrm{WM}\right.$ $\mathrm{V}+55,556 * \mathrm{CSF})) /(35,880 *(1-\mathrm{CSF}))$ and GABA-Corr $=$ $\left(\mathrm{GABA}^{*}(43,300 * \mathrm{GMV}+35,880 * \mathrm{WMV}+55,556 * \mathrm{CSF})\right) /$ $(35,880 *(1-\mathrm{CSF}))$.

To test for demographic differences between LS and HS groups, we used chi-square or independent sample $t$-tests. Differences between LS and HS groups in mPFC metabolite levels, as well as SNR, line width and CRLB, were established using multivariate analysis of variance (MANOVA) to control for multiple testing. These analyses also included BDI and STAI scores as covariates of no interest. Using logistic regression, we tested if resilience (CD-RISC scores) and GABA metabolite concentrations predicted SPQ group (low, high) membership. No assumptions of the logistic 
regression model were violated in the current samples. The analysis of multicollinearity revealed variance inflation factors of 3.42 and 4.71 for GABA_Corr and CD-RISC scores, respectively. For completeness, the regression model was repeated to investigate the effects of CD-RISC scores on glutamate metabolite concentrations and SPQ group. All analyses are conducted in $\mathrm{R}$ and reported at a significance level of $p<0.05$. There were no a priori hypotheses for other 1H-MRS metabolite levels as our focus was on GABA and Glu metabolite concentrations due to their key role in neuropathology of schizophrenia and in psychosis risk (Egerton et al. 2014; Marsman et al. 2013). For completeness, NAA, myo-inositol and creatine (commonly reported metabolite concentrations) are reported in Supplementary Materials.

\section{Results}

Due to the differing sample sizes for GABA and Glu metabolite concentrations resulting from quality control checks of the metabolite levels, we report the results separately. There was a significant positive strong correlation between the MRS measures of Glu and GABA in the participants in this study, $r=0.74, p<0.01$.

\section{MRS GABA metabolite concentrations}

Table 1 summarises the sociodemographic sample characteristics for the analysis of GABA metabolite concentrations in LS and HS groups. HS and LS groups were matched for gender, age and IQ but differed on all schizotypy measures, as intended by design. The low and high schizotypy groups differ significantly on measures of depression and trait anxiety. ANOVA revealed that HS groups had significantly lower GM, WM and CSF tissue volumes in the mPFC voxel compared to LS. Summaries of the quality check data for the GABA set by group are presented in Table 2. No significant differences between groups were detected for SN ratio, line width or CRLB. Using corrected metabolite concentration values and including depression and anxiety scores as covariates of no interest, ANOVA showed that the HS group $(\mathrm{M}=1.73, \mathrm{SD}=0.92)$ had significantly lower GABA-Corr levels than the LS group $(\mathrm{M}=2.36, \mathrm{SD}=0.62)$, $F(1,38)=5.18, p=0.03, \eta^{2}=0.09$ (Fig. 2a). Logistic regression showed that the interaction between GABA-Corr levels and CD-RISC scores (resilience) was a significant fit of the model, $\chi^{2}(3)=43.3, p<0.04$, Cox and Snell's $R^{2}=0.42$, Nagelkerke's $R^{2}=0.73$, and a significant predictor of SPQ group membership $(b=0.17, \mathrm{SE}=0.05, z=3.08, p<0.01)$. Figure 2 shows that participants with high GABA and resilience levels were significantly more likely to be in the low schizotypy group than those with low GABA and high resilience or high GABA and low resilience (95\% CI 1.07-1.34).

\section{MRS Glu metabolite concentrations}

Table 3 summarises the sociodemographic sample characteristics for the analysis of Glu metabolite concentrations in LS and HS groups. HS and LS groups were matched for gender, age and IQ but differed on all schizotypy measures, as intended by design. Similarly, to the results from the GABA analysis, HS groups showed significantly lower GM, WM and CSF tissue volumes compared to LS. Summaries of the quality check values for the Glu set based on group are presented in Table 2. Using glutamate-corrected metabolite concentration values and including STAI and BDI scores as covariates of no interest, ANOVA showed that the HS group $(\mathrm{M}=5.38, \mathrm{SD}=2.32)$ had significantly lower Glu-Corr levels than the LS group $(\mathrm{M}=6.71$, $\mathrm{SD}=2.28), F(1,49)=6.25, p=0.02, \eta^{2}=0.02$ (Fig. 2b). Linear regression showed no significant interaction effects between glutamate levels and CD-RISC (resilience) scores.
Table 1 Demographic summary of questionnaire and tissue maps across the HS and LS groups for GABA metabolite analysis

\begin{tabular}{lllrr}
\hline Characteristic & LS $(n=21)$ & HS $(n=19)$ & F/ $\chi^{2}$ & $p$ \\
\hline Gender (male/female) & $5 / 16$ & $6 / 13$ & 0.03 & 0.84 \\
Age (years) & $19.13(\mathrm{SD}=2.10)$ & $19.87(\mathrm{SD}=1.05)$ & 1.05 & 0.31 \\
IQ score & $93.95(\mathrm{SD}=9.13)$ & $92.89(\mathrm{SD}=9.69)$ & 0.12 & 0.72 \\
BDI-SF & $4.04(\mathrm{SD}=3.39)$ & $12.44(\mathrm{SD}=5.32)$ & 6.89 & $<0.01$ \\
STAI & $66.92(\mathrm{SD}=11.76)$ & $101.25(\mathrm{SD}=13.31)$ & 9.93 & $<0.01$ \\
SPQ total & $6.86(\mathrm{SD}=3.34)$ & $45.8(\mathrm{SD}=3.39)$ & 133.7 & $<0.01$ \\
SPQ cognitive perceptual factor & $2.67(\mathrm{SD}=2.13)$ & $20.8(\mathrm{SD}=3.96)$ & 335.53 & $<0.01$ \\
SPQ interpersonal factor & $3.14(\mathrm{SD}=2.26)$ & $20.1(\mathrm{SD}=3.81)$ & 299.32 & $<0.01$ \\
SPQ disorganised factor & $1.67(\mathrm{SD}=2.42)$ & $10.7(\mathrm{SD}=2.84)$ & 118.86 & $<0.01$ \\
Resilience & $61.5(\mathrm{SD}=13.7)$ & $74.1(\mathrm{SD}=13.0)$ & 8.91 & $<0.01$ \\
WM volume & $0.36(\mathrm{SD}=0.03)$ & $0.27(\mathrm{SD}=0.07)$ & 27.15 & $<0.01$ \\
GM volume & $0.42(\mathrm{SD}=0.03)$ & $0.29(\mathrm{SD}=0.11)$ & 24.17 & $<0.01$ \\
CSF volume & $0.16(\mathrm{SD}=0.02)$ & $0.12(\mathrm{SD}=0.03)$ & 19.73 & $<0.01$ \\
\hline
\end{tabular}


Table 2 Summary of quality measures for the GABA dataset and Glu dataset based on group and total sample

\begin{tabular}{lcccc}
\hline GABA & LS & HS & Total & Analysis (HS vs \\
& & & & LS), F statistics \\
\hline mPFC GABA & LS $(n=21)$ & HS $(n=19)$ & Total $(n=40)$ & \\
SN ratio & $53.23(\mathrm{SD}=9.98)$ & $51(\mathrm{SD}=5.24)$ & $52.23(\mathrm{SD}=7.82)$ & $0.005, p=0.34$ \\
Line width in Hz & $6.68(\mathrm{SD}=1.23)$ & $5.76(\mathrm{SD}=1.85)$ & $6.27(\mathrm{SD}=1.64)$ & $0.006, p=0.7$ \\
GABA CRLB (in \%) & $14.80(\mathrm{SD}=3.17)$ & $14.17(\mathrm{SD}=2.50)$ & $14.52(\mathrm{SD}=2.88)$ & $0.05, p=0.49$ \\
Glu & & & & \\
mPFC Glu & $\mathrm{LS}(n=26)$ & $\mathrm{HS}(n=25)$ & Total $(n=51)$ & \\
SN ratio & $52(\mathrm{SD}=9.04)$ & $51.4(\mathrm{SD}=6.64)$ & $51.70(\mathrm{SD}=7.88)$ & $0.005, p=0.78$ \\
Line width in Hz & $6.76(\mathrm{SD}=1.15)$ & $5.97(\mathrm{SD}=1.77)$ & $6.20(\mathrm{SD}=1.58)$ & $0.006, \mathrm{p}=0.6$ \\
Glu CRLB $($ in $\%)$ & $5.84(\mathrm{SD}=1.97)$ & $5.16(\mathrm{SD}=1.65)$ & $5.50(\mathrm{SD}=1.83)$ & $0.07, \mathrm{p}=0.18$ \\
\hline
\end{tabular}

\section{Discussion}

Using $1 \mathrm{H}-\mathrm{MRS}$, the present study found that relative to the LS group, the HS group had significantly lower mPFC GABA and glutamate metabolite concentrations. These results are in line with previous studies in schizophrenia populations which show that patients have lower mPFC GABA levels compared to healthy controls (Marsman et al. 2013; Öngür et al. 2010; Zhilei et al. 2015; Rowland et al. 2013). It should be noted however that our results are in contrast to the overall body of evidence in schizophrenia and risk populations as meta-analysis has reported no differences between patients and healthy controls for mPFC GABA metabolite concentrations (see Egerton
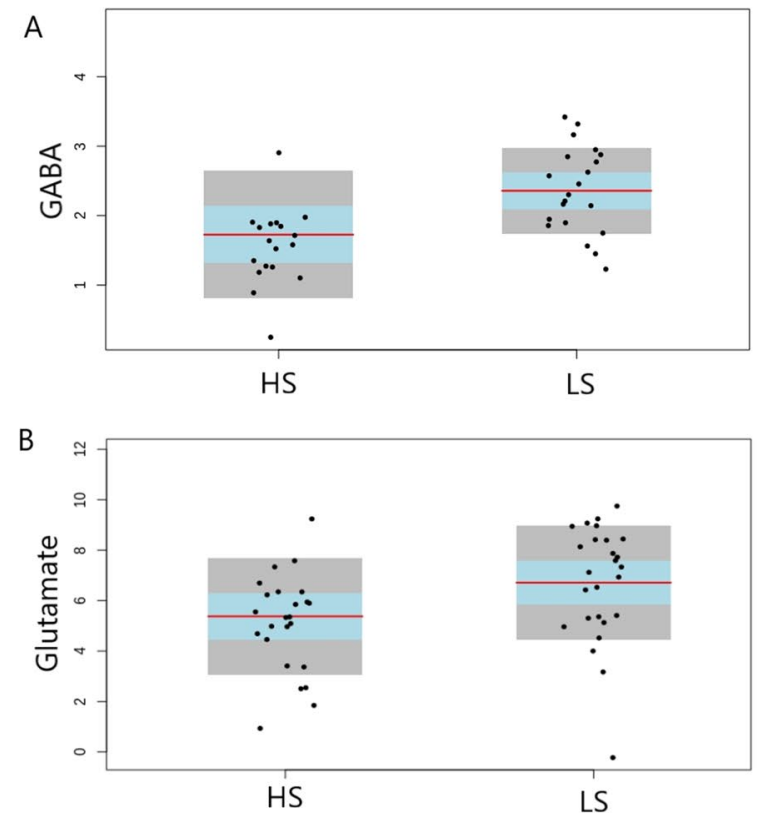

Fig. 2 a GABA-corrected levels and $\mathbf{b}$ Glu-corrected levels by SPQ groups. Error bars show the standard error of the mean. c Logistic regression interaction effect with GABA_Corr levels on the $\mathrm{x}$ axis

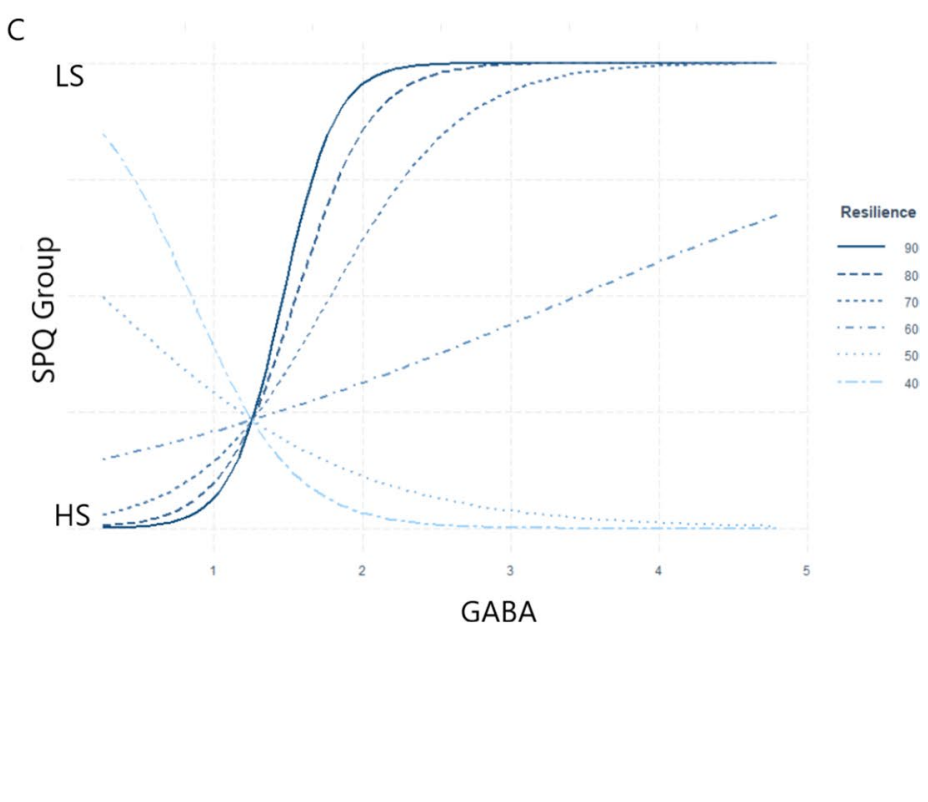

et al. (2017) for review). Studies investigating metabolite concentrations in individuals with a clinical high risk for psychosis, similarly to schizophrenia studies, have also found increased (Tayoshi et al. 2010), decreased (de la Fuente-Sandoval et al. 2016) or unaltered GABA levels in at-risk populations relative to healthy controls (Chen et al. 2014; Menschikov et al. 2016; Da Silva et al. 2019). Findings are further complicated by reports that in clinical high-risk individuals, mPFC GABA levels are negatively correlated with the severity of negative symptoms (Menschikov et al. 2016) and that unaffected siblings have significantly lower GABA levels compared with controls (Chen et al. 2014). One possible reason for differences found across different studies and study populations may be medication status, as unlike in schizophrenia patient

and SPQ group membership on the y axis; resilience is presented at the distributions of the resilience scores in the data 
Table 3 Demographic summary of questionnaire and tissue maps across the HS and LS groups

\begin{tabular}{lllrr}
\hline Characteristic & $\mathrm{LS}(n=26)$ & $\mathrm{HS}(n=25)$ & $\mathrm{t} / \chi^{2}$ & $p$ \\
\hline Gender (male/female) & $7 / 19$ & $8 / 17$ & 0.05 & 0.93 \\
Age (years) & $19.25(\mathrm{SD}=2.14)$ & $19.94(\mathrm{SD}=1.35)$ & 1.04 & 0.32 \\
IQ score & $95.1(\mathrm{SD}=9.16)$ & $93.8(\mathrm{SD}=9.80)$ & 0.26 & 0.61 \\
BDI-SF & $5.21(\mathrm{SD}=4.15)$ & $14.44(\mathrm{SD}=5.10)$ & 6.28 & $<0.01$ \\
STAI & $63.72(\mathrm{SD}=12.16)$ & $99.27(\mathrm{SD}=11.13)$ & 8.79 & $<0.01$ \\
SPQ total & $7.5(\mathrm{SD}=3.65)$ & $47.2(\mathrm{SD}=5.28)$ & 980.48 & $<0.01$ \\
SPQ cognitive perceptual factor & $2.69(\mathrm{SD}=1.95)$ & $20.7(\mathrm{SD}=5.06)$ & 284.21 & $<0.01$ \\
SPQ interpersonal factor & $3.65(\mathrm{SD}=2.67)$ & $21.3(\mathrm{SD}=4.20)$ & 324.13 & $<0.01$ \\
SPQ disorganised factor & $1.85(\mathrm{SD}=2.31)$ & $10.8(\mathrm{SD}=2.69)$ & 164.68 & $<0.01$ \\
Resilience & $73.58(\mathrm{SD}=12.93)$ & $58.36(\mathrm{SD}=14.23)$ & 15.93 & $<0.01$ \\
WM volume & $0.34(\mathrm{SD}=0.07)$ & $0.27(\mathrm{SD}=0.07)$ & 13.82 & $<0.01$ \\
GM volume & $0.41(\mathrm{SD}=0.06)$ & $0.29(\mathrm{SD}=0.11)$ & 20.03 & $<0.01$ \\
CSF volume & $0.16(\mathrm{SD}=0.02)$ & $0.12(\mathrm{SD}=0.03)$ & 20.91 & $<0.01$ \\
\hline
\end{tabular}

sample, participants with high schizotypy and/or a clinical risk for psychosis will not usually have been exposed to antipsychotic medication. Moreover, unlike studies in psychosis risk populations (clinical/genetic), studies in schizotypy populations can provide important information about neurobiological mechanisms that underlie schizophrenia phenotype across the continuum, in non-helpseeking individuals.

Despite inconsistent findings in humans, animal models of schizophrenia indicate that dysfunction of the GABAergic neurotransmitter system plays a major role in the pathophysiology of schizophrenia (Du and Grace 2013; Grace 2010). The MAM model of psychosis suggests a link between disrupted cortical GABAergic function and the dysregulation of hippocampal dopaminergic signalling (Grace 2010, 2016). The model posits that dopaminergic hyperactivity is an indirect consequence of the reduced number of parvalbumin inhibitory interneurons in the hippocampus and the PFC (Lodge and Grace 2008; Zhang et al. 2002). Parvalbumin interneurons contain and release GABA that inhibits, or limits, the activity of the neurons that provide output from the hippocampus and prefrontal cortex (Grace 2010). Indeed, studies revealed that MAM-treated rats show a selective loss of parvalbumincontaining interneurons in both the hippocampus and the prefrontal cortex (Lodge et al. 2009). The mPFC can regulate hippocampal and subcortical dopamine neuron activity via the nucleus reuniens of the thalamus (Grace 2010). Thus, the current finding of reduced mPFC GABA levels in high schizotypy participants is broadly consistent with diminished GABAergic regulation from the mPFC shown in the MAM model of psychosis, but whether this is due to a reduced density of parvalbumin interneurons (as shown in the animal model) cannot be established using MRS.

We also found that the interaction between resilience scores (the ability to cope with stress) and mPFC GABA metabolite concentrations predicted schizotypy group (low, high). Participants with both higher resilience and higher mPFC GABA metabolite concentrations were significantly more likely to be in the low schizotypy group. Whilst this finding is not straightforward to interpret, it suggests that both high resilience and mPFC GABA levels, and how these variables interact, may protect individuals from psychoticlike experiences. This result is broadly consistent with preclinical models of psychosis that show that environmental stress affects the GABAergic system in humans (Zhang et al. 2010; Guidotti et al. 2011), consequently increasing risk for psychosis (Zimmerman et al. 2013). However, the association between resilience levels and mPFC GABA metabolite concentrations did not differ significantly in low and high schizotypy groups. Thus, the relationship between resilience and mPFC GABA levels, and how this differs in people who experience psychosis-like symptoms, remains unclear.

The results are consistent with models linking stress sensitivity with the experience of psychotic symptoms (Barrantes-Vidal et al. 2013). Previous work has shown that stressful situations and social stress are associated with momentary psychotic-like and paranoid symptoms for those high in positive schizotypy, but not those low in positive schizotypy (Barrantes-Vidal et al. 2013) and that the interaction between schizotypy traits and stress significantly predicted reasoning biases (Le et al. 2019). Studies have also shown that baseline cortisol levels in adolescents with schizotypal symptoms predict severity of their schizotypal symptoms later in life (Walker et al. 2010) suggesting further that stress interacts with schizotypal traits.

Cortical glutamate levels were also found to be significantly lower in high compared to low schizotypy individuals, a finding consistent with some previous studies in patients with schizophrenia (Marsman et al. 2013; Öngür et al. 2010; Goto et al. 2009). However, as with studies of GABA metabolite concentrations, increased cortical glutamate has 
also been reported in patients with schizophrenia (Choe et al. 1996; Rüsch et al. 2008; Moore et al. 2006). Mixed results are also reported in studies investigating clinical high-risk samples, where decreased (Natsubori et al. 2014) and increased cortical glutamate levels have been reported (Stone et al. 2009). Animal models of psychosis generally posit a role for increased glutamate levels, particularly in medial temporal lobe regions, that leads to reduced hippocampal volume (Lieberman et al. 2018) and increased subiculum output to the ventral striatum via glutamatergic pathways (Moore et al. 2006; Lodge and Grace 2011). However, decreased glutamate levels have been reported in the mPFC in MRS studies of patients with schizophrenia and CHR samples (Marsman et al. 2013), consistent with the current findings in high schizotypy individuals. The glutamatergic system is believed to affect synaptic plasticity and cortical microcircuitry, in particular NMDA receptor signalling (Harrison and Weinberger 2005). NMDA receptor antagonists, such as ketamine and phencyclidine (PCP) which reduce glutamatergic signalling, induce symptoms that mimic the positive and negative symptoms seen in schizophrenia (Adams and Moghaddam 1998; Krystal et al. 1994; Moghaddam et al. 1997). Injection of these NMDA receptor antagonists leads to decreased glutamate levels (Marsman et al. 2013; Moghaddam et al. 1997; Rowland et al. 2005) and animal studies show that the absence of NMDA receptor subunits can cause alterations at the molecular and behavioural level and produce schizophrenia-like symptoms (Marsman et al. 2013).

Changes in cortical inhibitory and excitatory signalling may result in a loss of synchronous cortical activity (Lisman et al. 2008; Lewis et al. 2012) and underlie the behavioural deficits commonly reported in schizophrenia and psychosis risk populations (Lisman et al. 2008; Lewis et al. 2012), such as emotional processing (Keefe et al. 2005) and social cognition (Kozhuharova et al. 2019). In terms of schizotypy as a personality dimension, alterations in cortical excitatory and inhibitory neurotransmission are likely to have implications for cognition, perception and interpersonal skills (which may form a continuum with the florid abnormalities seen in psychiatric disorders). Indeed, a recent systematic review conducted by our research group reported that high schizotypy (and clinical at-risk populations) show a tendency towards increased activity in frontal cortex during various emotional and social cognition tasks (Kozhuharova et al. 2019). There is also an established literature reporting behavioural and functional alterations in cognition/executive function and the underlying neural substrate in people with high schizotypal traits (Ettinger et al. 2015; Nelson et al. 2013; Kwapil and Barrantes-Vidal 2015). The current findings therefore have implications for cognitive and behavioural performance associated with high schizotypal traits. Animal model studies show that a functional loss of
GABA-mediated inhibition diminishes gamma oscillations (Lodge et al. 2009) and impairs cognitive function (Enomoto et al. 2011; Gruber et al. 2010). There is also preliminary evidence that glutamate also affects cognitive performance (Ohrmann et al. 2007; Bustillo et al. 2011; Shirayama et al. 2010; Merritt et al. 2013). Schizotypy traits are also associated with impaired cognitive functioning (Cochrane and Reynolds 2012; Bedwell et al. 2009; Völter et al. 2012) and the current findings suggest that abnormal inhibitory and excitatory metabolite levels might be a mechanism that affects cognitive performance across the psychosis continuum.

Although much more work is needed to investigate the relationship between cortical GABA/glutamate signalling and behavioural measures, one previous study investigating glutamate levels in individuals with high positive schizotypy reports that glutamate levels were negatively associated with the degree of activation to emotional pictures in the striatum and the mPFC (Modinos et al. 2017). Although we acquired no behavioural measures here, future work should examine the relationship between GABA/glutamate and cognitive function, affective processing and emotion regulation in high schizotypy samples.

A limitation of 1H-MRS is that it measures total GABA concentrations within a relatively large voxel, which is determined a priori, and cannot discriminate between GABA levels in different cell types. This limits the application of $1 \mathrm{H}-\mathrm{MRS}$ in addressing the cell- and network-specific GABA abnormalities hypothesised to occur in schizophrenia and psychosis risk (Lewis et al. 2012). For this reason, the current findings cannot inform on the specific mechanisms that might lead to reduced GABA and glutamate levels and we cannot test whether these results are due to reduced GAD67 or reduced density of parvalbumin interneurons, as suggested by animal models. The $1 \mathrm{H}-\mathrm{MRS}$ GABA signal may reflect the entire GABA content of the voxel (that is, intracellular and extracellular, and involved in metabolism or neurotransmission). Recent work argues that the 1H-MRS GABA signal predominantly relates to extracellular, extra-synaptic GABA providing tonic inhibitory tone, rather than GABA involved in phasic synaptic neurotransmission (Stagg 2014). Theoretically, the 1H-MRS GABA signal should therefore be sensitive to GAD67 reduction, but we could not test this in the current study. Pharmacologically induced alterations in synaptic GABA may be more sensitively imaged with positron emission tomography (PET, Egerton et al. 2017). In future, combination of this approach with $1 \mathrm{H}-\mathrm{MRS}$ in the same subjects, and potentially during the same scanning session on combined PET-magnetic resonance platforms, might investigate dysfunction of synaptic versus non-synaptic GABA and glutamate in schizophrenia.

Whilst mood and anxiety disorders can affect neurometabolite levels, and the low and high schizotypy groups 
did differ significantly on measures of depression and trait anxiety, significant group differences for both GABA and glutamate metabolite concentrations were observed after STAI and BDI scores were added as covariates of no interest. Affective symptoms are however common in people with schizophrenia (Huppert et al. 2001), clinical high risk (Fusar-poli et al. 2014) and in high schizotypy samples (Lewandowski et al. 2006), and are considered to be part of the schizophrenia phenotype (Van Os and Reininghaus 2016; Upthegrove et al. 2017). In practice, the distinction between schizotypal/schizophrenia symptomatology and comorbid depressive/anxious traits is difficult to establish and there is little evidence to instruct diagnostic separation (Malhi et al. 2008). A further limitation of the study is that we did not record any family history of neuropsychiatric disorders and, although self-report data was sought and presented (see Supplementary Material section), we did not conduct urine drug screening to objectively test recent substance abuse. However, the latter is not a common practice in MRI schizotypy studies (Modinos et al. 2010; Ettinger et al. 2012; Wang et al. 2015). Moreover, whilst analyses covaried for anxiety and depression scores, we were unable to conduct a full clinical assessment in our high and low schizotypy groups. Thus, it was not possible to delineate whether schizotypal symptoms, particularly negative and interpersonal symptoms, were primary to a mood and/or anxiety disorder. Future studies should conduct a detailed clinical assessment to better characterise symptom profiles in high schizotypy participants.

A final limitation of the sample is that only the SPQ total score was used to define groups and future work should aim to delineate the MRS correlates of the specific schizotypal subfactors further. Indeed, much of the work in experimental animals discussed earlier pertains to psychosis and psychotic-like symptomology, rather than the schizophrenia syndrome. However, our approach follows the design of many clinical studies in schizophrenia patients, who experience a constellation of negative, positive and cognitive symptoms. Future work should use updated versions of the SPQ with a Likert scale rather than a dichotomous (true/ false) response. This would allow for a better measure of trait severity.

In conclusion, the current study utilised MRS methods to investigate GABA and glutamate levels in individuals with high and low schizotypy levels. In line with predictions from animal and post-mortem studies of schizophrenia, the current study is the first to report reduced levels of both GABA and glutamate metabolite concentrations in high schizotypy individuals compared to low schizotypy. Furthermore, the current work supports the role of stress sensitivity in the development of schizophrenia-like symptoms. Whilst our results suggest that individuals with high schizotypal personality traits show similar changes in mPFC GABA and glutamate metabolite concentrations to those reported in some studies in schizophrenia and psychosis risk cohorts, they are in contrast to meta-analysis findings in these groups. These findings also suggest that changes in prefrontal GABA levels may be related to stress sensitivity, although more work is needed to better understand the role of stress in this relationship. Whilst the study design does not allow us to draw any conclusions about risk factors for schizophrenia, the findings provide support for the notion of a neurobiological psychosis continuum, and for the role of stress sensitivity in the development of schizophrenia-like symptoms.

Supplementary Information The online version contains supplementary material available at https://doi.org/10.1007/s00213-021-05867-y.

Acknowledgements This work was undertaken as part of a PhD scholarship with the Department of Psychology, University of Roehampton, awarded to the first author. AOD acknowledges support by the Swiss National Foundation (SNF PZ00P3_167952) and the Krembil Foundation.

\section{Declarations}

Competing interests The authors declare no competing interests.

Open Access This article is licensed under a Creative Commons Attribution 4.0 International License, which permits use, sharing, adaptation, distribution and reproduction in any medium or format, as long as you give appropriate credit to the original author(s) and the source, provide a link to the Creative Commons licence, and indicate if changes were made. The images or other third party material in this article are included in the article's Creative Commons licence, unless indicated otherwise in a credit line to the material. If material is not included in the article's Creative Commons licence and your intended use is not permitted by statutory regulation or exceeds the permitted use, you will need to obtain permission directly from the copyright holder. To view a copy of this licence, visit http://creativecommons.org/licenses/by/4.0/.

\section{References}

Adams B, Moghaddam B (1998) Corticolimbic dopamine neurotransmission is temporally dissociated from the cognitive and locomotor effects of phencyclidine. J Neurosci 18(14):5545-5554

Akbarian S, Kim JJ, Potkin SG, Hagman JO, Tafazzoli A, Bunney WE, Jones EG (1995) Gene expression for glutamic acid decarboxylase is reduced without loss of neurons in prefrontal cortex of schizophrenics. Arch Gen Psychiatry 52(4):258-266

Barrantes-Vidal N, Chun CA, Myin-Germeys I, Kwapil TR (2013) Psychometric schizotypy predicts psychotic-like, paranoid, and negative symptoms in daily life. J Abnorm Psychol 122(4):1077

Barrantes-Vidal N, Grant P, Kwapil T (2014) The role of schizotypy in the study of the etiology of schizophrenia spectrum disorders. Schizophr Bull 41(2):S408-S441

Becker A, Peters B, Schroeder H, Mann T, Guether G, Grecksch G (2003) Ketamine-induced changes in rat behaviour: a possible animal model of schizophrenia. Prog Neuropsychopharmacol Biol Psychiatry 27(4):687-700 
Bedwell JS, Kamath V, Compton MT (2009) The relationship between interview-based schizotypal personality dimension scores and thecontinuous performance test. Schizophr Res 108(1-3):158-162

Bustillo JR, Chen H, Gasparovic C, Mullins P, Caprihan A, Qualls C ... \& Posse $S$ (2011) Glutamate as a marker of cognitive function in schizophrenia: a proton spectroscopic imaging study at 4 Tesla. Biol Psychiatry 69(1):19-27

Campbell-Sills L, Stein M (2007) Psychometric analysis and refinement of the Connor-Davidson resilience scale (CD-RISC): validation of a 10-item measure of resilience. J Trauma Stress 20(6):1019-1028

Chen C, Stanford A, Mao X, Abi-Dargham A, Shungu D, Lisanby S, Kegeoes L (2014) GABA level, gamma oscillation, and working memory performance in schizophrenia. NeuroImage Clinical 4:531-539

Choe B, Suh T, Shinn K, Lee C, Lee C, Paik I (1996) Observation of metabolic changes in chronic schizophrenia after neuroleptic treatment by in vivo hydrogen magnetic resonance spectroscopy. Invest Radiol 31(6):345-352

Cochrane NJ, Reynolds EC (2012) Calcium phosphopeptides-mechanisms of action and evidence for clinical efficacy. Adv Dent Res 24(2):41-47

Da Silva T, Hafizi S, Rusjan P, Houle S, Wilson A, Prce I, Mizrahi R (2019) GABA levels and TSPO expression in people at clinical high risk for psychosis and healthy volunteers: a PET-MRS study. J Psychiatry Neurosci 44(2):111

de la Fuente-Sandoval C, Reyes-Madrigal F, Mao X, Leon-Ortiz P, Rodriguez-Mayoral O, Solis-Vivanco R, Shungu D (2016) Cortico-striatal GABAergic and glutamatergic dysregulations in subjects at ultra-high risk for psychosis investigated with proton magnetic resonance spectroscopy. Int J Neuropsychopharmacol 19(3): 105

Deslauriers J, Larouche A, Sarret P, Grignon S (2013) Combination of prenatal immune challenge and restraint stress affects prepulse inhibition and dopaminergic/GABAergic markers. Prog Neuropsychopharmacol Biol Psychiatry 45:156-164

Du Y, Grace A (2013) Peripubertal diazepam administration prevents the emergence of dopamine system hyperresponsivity in the MAM developmental disruption model of schizophrenia. Neuropsychopharmacology 38(10):1881-1888

Egerton A, Stone JM, Chaddock CA, Barker GJ, Bonoldi I, Howard RM, Merritt K, Allen P, Howes OD, Murray RM, McLean MA, Lythgoe DJ, O'Gorman RL, McGuire PK (2014) Relationship between brain glutamate levels and clinical outcome in individuals at ultra high risk of psychosis. Neuropsychopharmacology 39(12):2891-2899

Egerton A, Modinos G, Ferrera D, McGuire P (2017) Neuroimaging studies of GABA in schizophrenia: a systematic review with metaanalysis. Transl Psychiatry 7(6):e1147-e1147

Enomoto T, Maric TT, Floresco SB (2011) Reducing prefrontal gamma-aminobutyric acid activity induces cognitive, behavioral, and dopaminergic abnormalities that resemble schizophrenia. Biol Psychiat 69(5):432-441

Ettinger U, Meyhofer I, Steffens M, Wagner M, Koutsouleris N (2014) Genetics, cognition, and neurobiology of schizotypal personality: a review of the overlap with schizophrenia. Front Psych 5:18

Ettinger U, Williams SC, Meisenzahl EM, Möller HJ, Kumari V, Koutsouleris N (2012) Association between brain structure and psychometric schizotypy in healthy individuals. World J Biol Psychiatry 13(7):544-549

Ettinger U, Mohr C, Gooding DC, Cohen AS, Rapp A, Haenschel C, Park S (2015) Cognition and brain function in schizotypy: a selective review. Schizophr Bull 41(suppl_2):S417-S426

Fischer D, Fick C (1993) Measuring social desirability: short forms of the Marlowe-Crowne social desirability scale. Educ Psychol Measur 53(2):417-424
Furlanetto LM, Mendlowicz MV, Bueno JR (2005) The validity of the Beck Depression Inventory-Short Form as a screening and diagnostic instrument for moderate and severe depression in medical inpatients. J Affect Disord 86(1):87-91

Fusar-Poli P, Nelson B, Valmaggia L, Yung AR, McGuire PK (2014) Comorbid depressive and anxiety disorders in 509 individuals with an at-risk mental state: impact on psychopathology and transition to psychosis. Schizophr Bull 40(1):120-131

Gasparovic C, Song T, Devier D, Bockholt HJ, Caprihan A, Mullins PG, Posse S, Jung RE, Morrison LA (2006) Use of tissue water as a concentration reference for proton spectroscopic imaging. Magn Reson Med 55(6):1219-1226

Gastambide F, Cotel M, Gilmour G, O’Neill M, Robbins T, Tricklebank M (2012) Selective remediation of reversal learning deficits in the neurodevelopmental MAM model of schizophrenia by a novel mGlu5 positive allosteric modulator. Neuropsychopharamacology 37(4):1057-1066

Godlewska B, Near J, Cowen P (2015) Neurochemistry of major depression: a study using magnetic resonance spectroscopy. Psychopharmacology 232(3):501-507

Goto N, Yoshimura R, Moriya J, Kakeda S, Ueda N, Ikenouchi-Sugita A, Nakamura J (2009) Reduction of brain gamma-aminobutyric acid (GABA) concentrations in early-stage schizophrenia patients: 3T Proton MRS study. Schizophr Res 112(1-3):192-193

Goto Y, Grace A (2006) Alterations in medial prefrontal cortical activity and plasticity in rats with disruption of cortical development. Biol Psychiat 60(11):1259-1267

Grace A (2010) Ventral hippocampus, interneurons, and schizophrenia: a new understanding of the pathophysiology of schizophrenia and its implications for treatment and prevention. Curr Dir Psychol Sci 19:232-237

Grace A (2016) Dysregulation of the dopamine system in the pathophysiology of schizophrenia and depression. Nat Rev Neurosci 17:524-532

Grös DF, Antony MM, Simms LJ, McCabe RE (2007) Psychometric properties of the state-trait inventory for cognitive and somatic anxiety (STICSA): comparison to the state-trait anxiety inventory (STAI). Psychol Assess 19(4):369

Gruber AJ, Calhoon GG, Shusterman I, Schoenbaum G, Roesch MR, O'Donnell P (2010) More is less: a disinhibited prefrontal cortex impairs cognitive flexibility. J Neurosci 30(50):17102-17110

Guidotti A, Auta J, Chen Y, Davis J, Dong E, Gavin D, Sharma R (2011) Epigenetic GABAergic targets in schizophrenia and bipolar disorder. Neuropharmacology 60(7-8):1007-1016

Harrison P, Weinberger D (2005) Schizophrenia genes, gene expression, and neuropathology: on the matter of their convergence. Mol Psychiatry 10(1):40-68

Huppert JD, Weiss KA, Lim R, Pratt S, Smith TE (2001) Quality of life in schizophrenia: contributions of anxiety and depression. Schizophr Res 51(2-3):171-180

Huster R, Westerhausen R, Kreuder F, Schweiger E, Wittling W (2007) Morphologic asymmetry of the human anterior cingulate cortex. Neuroimage 34(3):888-895

Keefe R, Eesley C, Poe M (2005) Defining a cognitive function decrement in schizophrenia. Biol Psychiat 57(6):688-691

Kozhuharova P, Saviola F, Ettinger U, Allen P (2019) Neural correlates of social cognition in populations at risk of psychosis: a systematic review. Neurosci Biobehav Rev 108:94-111

Krystal JH, Karper LP, Seibyl JP, Freeman GK, Delaney R, Bremner JD, ... \& Charney DS (1994) Subanesthetic effects of the noncompetitive NMDA antagonist, ketamine, in humans: psychotomimetic, perceptual, cognitive, and neuroendocrine responses. Arch Gen Psychiatry 51(3):199-214

Kwapil TR, Barrantes-Vidal N (2015) Schizotypy: looking back and moving forward. Schizophr Bull 41(suppl_2):S366-S373 
Le TL, Cohen AS, Allred S, Pham C, Lewis S, Barkus E (2019) Stress and cognitive biases in schizotypy: a two-site study of bias against disconfirmatory evidence and jumping to conclusions. Eur Psychiatry 62:20-27

Lewandowski KE, Barrantes-Vidal N, Nelson-Gray RO, Clancy C, Kepley HO, Kwapil TR (2006) Anxiety and depression symptoms in psychometrically identified schizotypy. Schizophr Res 83(2-3):225-235

Lewis D, Curley A, Glausier J, Volk D (2012) Cortical parvalbumin interneurons and cognitive dysfunction in schizophrenia. Trends Neurosci 35(1):57-67

Lewis D, Hashimoto T, Volk D (2005) Cortical inhibitory neurons and schizophrenia. Nat Rev Neurosci 6:312-324

Lieberman JA, Girgis RR, Brucato G, Moore H, Provenzano F, Kegeles L, ... \& Schobel SA (2018) Hippocampal dysfunction in the pathophysiology of schizophrenia: a selective review and hypothesis for early detection and intervention. Mol Psychiatry 23(8): 1764-1772

Linscott R, Van Os J (2013) An updated and conservative systematic review and meta-analysis of epidemiological evidence on psychotic experiences in children and adults: on the pathway from proneness to persistence to dimensional expression across mental disorders. Psychol Med 43(6):1133-1149

Lisman J, Coyle J, Green R, Javitt D, Benes F, Heckers S, Grace A (2008) Circuit-based framework for understanding neurotransmitter and risk gene interactions in schizophrenia. Trends Neurosci 31(5):234-242

Lodge DJ, Grace AA (2011) Hippocampal dysregulation of dopamine system function and the pathophysiology of schizophrenia. Trends Pharmacol Sci 32(9):507-513

Lodge D, Grace A (2008) Hippocampal dysfunction and disruption of dopamine system regulation in an animal model of schizophrenia. Neurotox Res 14(2-3):97-104

Lodge D, Behrens M, Grace A (2009) A loss of parvalbumin-containing interneurons is associated with diminished oscillatory activity in an animal model of schizophrenia. J Neurosci 29(8):2344-2354

Mailly P, Aliane V, Groenewegen H, Haber S, Deniau J (2013) The rat prefrontostriatal system analyzed in 3D: evidence for multiple interacting functional units. J Neurosci 33(13):5718-5727

Malhi GS, Green M, Fagiolini A, Peselow ED, Kumari V (2008) Schizoaffective disorder: diagnostic issues and future recommendations. Bipolar Disord 10(1p2):215-230

Marsman A, van den Heuvel M, Klomp D, Kahn R, Luijten P, Hulshoff Pol H (2013) Glutamate in schizophrenia: a focused review and meta-analysis of 1H-MRS studies. Schizophr Bull 39(1):120-129

Menschikov P, Semenova N, Ublinskiy M, Akhadov T, Keshishyan R, Lebedeva I, Varfolomeev S (2016) 1 H-MRS and MEGA-PRESS pulse sequence in the study of balance of inhibitory and excitatory neurotransmitters in the human brain of ultra-high risk of schizophrenia patients. Dokl Biochem Biophys 468(1):168-172

Merritt K, Egerton A, Kempton MJ, Taylor MJ, McGuire PK (2016) Nature of glutamate alterations in schizophrenia: a meta-analysis of proton magnetic resonance spectroscopy studies. JAMA Psychiat 73(7):665-674

Merritt K, McGuire P, Egerton A (2013) Relationship between glutamate dysfunction and symptoms and cognitive function in psychosis. Front Psych 4:151

Mlynárik K, Gambarota G, Frenkel H, Gruetter R (2006) Localized short-echo-time proton MR spectroscopy with full signal-intensity acquisition. Magn Reson Med 56(5):965-970

Modinos G, McLaughlin A, Egerton A, McMullen K, Kumari V, Barker G, Williams S (2017) Corticolimbic hyper-response to emotion and glutamatergic function in people with high schizotypy: a multimodal fMRI-MRS study. Transl Psychiatry 7(4):e1083-e1083
Modinos G, Mechelli A, Ormel J, Groenewold NA, Aleman A, McGuire PK (2010) Schizotypy and brain structure: a voxel-based morphometry study. Psychol Med 40(9):1423-1431

Modinos G, Simsek F, Horder J, Bossong M, Bonoldi I, Azis M, Howes $\mathrm{O}$ (2018) Cortical GABA in subjects at ultra-high risk of psychosis: relationship to negative prodromal symptoms. Int J Neuropsychopharmacol 21(2):114-119

Moghaddam B, Adams B, Verma A, Daly D (1997) Activation of glutamatergic neurotransmission by ketamine: a novel step in the pathway from NMDA receptor blockade to dopaminergic and cognitive disruptions associated with the prefrontal cortex. J Neurosci 17(8):2921-2927

Moore H, Jentsch J, Ghajarnia M, Geyer M (2006) A neurobehavioral systems analysis of adult rats exposed to methylazoxymethanol acetate on E17: implications for the neuropathology of schizophrenia. Biol Psychiat 60(3):253-264

Natsubori T, Inoue H, Abe O, Takano Y, Iwashiro N, Aoki Y, Sasaki $\mathrm{H}$ (2014) Reduced frontal glutamate + glutamine and N-acetylaspartate levels in patients with chronic schizophrenia but not in those at clinical high risk for psychosis or with first-episode schizophrenia. Schizophr Bull 40(5):1128-1139

Nelson MT, Seal ML, Pantelis C, Phillips LJ (2013) Evidence of a dimensional relationship between schizotypy and schizophrenia: a systematic review. Neurosci Biobehav Rev 37(3):317-327

Ohrmann P, Siegmund A, Suslow T, Pedersen A, Spitzberg K, Kersting A, ... \& Pfleiderer B (2007) Cognitive impairment and in vivo metabolites in first-episode neuroleptic-naive and chronic medicated schizophrenic patients: a proton magnetic resonance spectroscopy study. J Psychiatr Res 41(8):625-634

Öngür D, Prescot A, McCarthy J, Cohen B, Renshaw P (2010) Elevated gamma-aminobutyric acid levels in chronic schizophrenia. Biol Psychiat 68(7):667-670

Powell SB, Sejnowski TJ, Behrens MM (2012) Behavioral and neurochemical consequences of cortical oxidative stress on parvalbumininterneuron maturation in rodent models of schizophrenia. Neuropharmacology 62(3):1322-1331

Raine A (1991) The SPQ: a scale for the assessment of schizotypal personality based on DSM-III-R criteria. Schizophr Bull 17(4):555-565

Rowland LM, Astur RS, Jung RE, Bustillo JR, Lauriello J, Yeo RA (2005) Selective cognitive impairments associated with NMDA receptor blockade in humans. Neuropsychopharmacology 30(3):633-639

Rowland L, Kontson K, West J, Edden R, Zhu H, Wijtenburg S, Barker P (2013) In vivo measurements of glutamate, GABA, and NAAG in schizophrenia. Schizophr Bull 39(5):1096-1104

Rüsch N, van Elsl L, Valerius G, Büchert M, Thiel T, Ebert D, Olbrich $\mathrm{H}$ (2008) Neurochemical and structural correlates of executive dysfunction in schizophrenia. Schizophr Res 99(1-3):155-163

Shirayama Y, Obata T, Matsuzawa D, Nonaka H, Kanazawa Y, Yoshitome E, ... \& Iyo M (2010) Specific metabolites in the medial prefrontal cortex are associated with the neurocognitive deficits in schizophrenia: a preliminary study. Neuroimage 49(3):2783-2790

Srinivasn R, Cunningham C, Chen A, Vigneron D, Hurd R, Nelson S, Pelletier D (2006) TE-averaged two-dimensional proton spectroscopic imaging of glutamate at $3 \mathrm{~T}$. Neuroimage 30(4):1171-1178

Stagg C (2014) Magnetic resonance spectroscopy as a tool to study the role of GABA in motor-cortical plasticity. Neuroimage 86:19-27

Stone DJ, Walsh JP, Sebro R, Stevens R, Pantazopolous H, Benes FM (2001) Effects of pre-and postnatal corticosterone exposure on the rat hippocampal GABA system. Hippocampus 11(5):492-507

Stone J, Day F, Tsagaraki H, Valli I, McLean M, Lythgoe D, McGuire $\mathrm{P}$ (2009) Glutamate dysfunction in people with prodromal symptoms of psychosis: relationship to gray matter volume. Biol Psychiat 66(6):533-539 
Tayoshi S, Nakataki M, Sumitani S, Taniguchi K, Shibuya-Tayoshi S, Numala S, Ohmori T (2010) GABA concentration in schizophrenia patients and the effects of antipsychotic medication: a proton magnetic resonance spectroscopy study. Schizophr Res 117(1):83-91

Upthegrove R, Marwaha S, Birchwood M (2017) Depression and schizophrenia: cause, consequence, or trans-diagnostic issue? Schizophr Bull 43(2):240-244

Van Os J, Reininghaus U (2016) Psychosis as a transdiagnostic and extended phenotype in the general population. World Psychiatry 15(2):118-124

Völter C, Strobach T, Aichert DS, Wöstmann N, Costa A, Möller HJ, Schubert T, Ettinger U (2012) Schizotypy and behavioural adjustment and the role of neuroticism. PloS One 7(2):e30078

Walker EF, Brennan PA, Esterberg M, Brasfield J, Pearce B, Compton MT (2010) Longitudinal changes in cortisol secretion and conversion to psychosis in at-risk youth. J Abnorm Psychol 119(2):401

Zhang T, Hellstrom I, Bagot R, Wen X, Diorio J, Meaney M (2010) Maternal care and DNA methylation of a glutamic acid decarboxylase 1 promoter in rat hippocampus. J Neuroscience 30(39):131-213
Zhang Y, Brady M, Smith S (2001) Segmentation of brain MR images through a hidden Markov random field model and the expectationmaximization algorithm. IEEE Trans Med Imaging 20(1):45-57

Wang Y, Yan C, Yin DZ, Fan MX, Cheung EF, Pantelis C, Chan RC (2015) Neurobiological changes of schizotypy: evidence from both volume-based morphometric analysis and resting-state functional connectivity. Schizophr Bull 41(suppl_2):S444-S454

Zhang Z, Sun J, Reynolds GP (2002) A selective reduction in the relative density of parvalbumin-immunoreactive neurons in the hippocampus in schizophrenia patients. Chin Med J 115(6):819-823

Zhilei Y, Yajing Z, Zhenhua S, Li M, Jianye T, Yingchan W, Dengtang L (2015) Comparison of the density of gamma-aminobutyric acid in the ventromedial prefrontal cortex of patients with firstepisode psychosis and healthy controls. Shanghai Arch Psychiatry 27(6):341

Zimmerman EC, Bellaire M, Ewing SG, Grace AA (2013) Abnormal stress responsivity in a rodent developmental disruption model of schizophrenia. Neuropsychopharmacology 38(11):2131-2139

Publisher's note Springer Nature remains neutral with regard to jurisdictional claims in published maps and institutional affiliations. 